Math. Struct. in Comp. Science (2001), vol. 11, p. 1. Printed in the United Kingdom

(c) 2001 Cambridge University Press

\title{
Foreword
}

\section{Theory and applications of explicit substitutions: Introduction}

This special issue of Mathematical Structures in Computer Science is dedicated to the theory and applications of explicit substitutions, which have attracted a growing community of researchers in the last decade, especially in the study of explicit substitutions as a means of bridging the gap between theory and practice in the implementation of programming languages, as well as theorem provers and proof checkers.

Such implementations typically rely on formal calculi defined using implicit substitution operations that are left at the meta-level, so that they need to turn these meta-level operations into efficient executable code, and this is often fairly intricate and distant from the formal calculi. This causes a significant gap between theory and practice.

Explicit substitutions considerably reduce this gap by bringing the meta-level operations down to the object-level calculus - where they are represented explicitly - allowing us in this way to give formal and robust models for the techniques actually used in implementations, and providing at the same time a more flexible tool for controlling the intermediate steps of evaluation.

All the papers in this issue were invited on the basis of their quality and relevance to the domain, and subjected to the refereeing process of MSCS. Most of them are substantially expanded and revised versions of work originally presented at Westapp' 98 and Westapp'99, the first and second 'Workshop on Explicit Substitutions: Theory and Applications to Programs and Proofs', which were held in conjunction with RTA'98 in Tsukuba, Japan, and with Floc'99 in Trento, Italy, respectively.

As guest editor, I would like to express my warm thanks both to the authors, for their high-quality contributions to this special issue, and to the referees, whose scientific role was essential in improving the presentation of these contributions.

Delia Kesner - guest editor Laboratoire de Recherche en Informatique,

Université Paris-Sud 